\title{
Über Lithiumamalgame.
}

\author{
Von \\ G. J. Żukowsky. ${ }^{1}$
}

Mit 3 Figuren im Text.

Die Legierungen der Alkalimetalle mit Quecksilber waren Gegenstand vielfacher Untersuchungen, und zwar sind die Natrium-, Kalium- und Cäsiumamalgame am eingehendsten studiert worden. Die Lithiumamalgame dagegen wurden bis jetzt fast gar nicht behandelt, und die geringe Anzahl der Arbeiten, die sich mit diesem binären Systeme beschäftigten, haben eher einen präparativen Charakter als dafs sie ein systematisches Studium bedeuteten.

KeRp, Bötger, WINTER und IGENA ${ }^{2}$ machten beim Studium der Amalgame der Alkali- und Erdalkalimetalle den Versuch, ein Lithiumamalgam zu gewinnen; es gelang ihnen jedoch nur eine einzige Verbindung zu isolieren, welcher sie die Zusammensetzung $\mathrm{LiHg}_{5}$ zuschrieben.

Ferner geben Guxtz und FereE ${ }^{3}$ die Existenz derselben Verbindung $\operatorname{LiHg}_{5}$ an.

Etwas ausführlicher ist dieses Metallpaar von $\mathrm{MEY}^{4}$ untersucht worden, welcher auf Grund der Änderungen der spezifischen Volumina der Legierungen in Abhängigkeit ron ihrer Zusammensetzung zu dem Schluls gelangte, dafs vier bestimmte Verbindungen: $\mathrm{LiHg}_{5}$, $\mathrm{LiHg}_{3}, \mathrm{LiHg}$ und $\mathrm{Li}_{3} \mathrm{Hg}$ existieren.

Alle diese Arbeiten charakterisieren dieses System nicht ausreichend; daher erschien es von Interesse auf die gegenseitigen Beziehungen zwischen Lithium und Quecksilber näher einzugehen.

Bei der Betrachtung der Schmelzdiagramme der binären Systeme aus Quecksilber und den anderen Alkalimetallen ist besonders der Umstand bemerkenswert, dafs alle diese Systeme die Fähigkeit

${ }^{1}$ Aus den Ber. d. Polytechn. Institut St. Petersb. 14 (1910), 655 ins Deutsche übertragen von I. Pingrer Berlin.

2 Kerp, Bötaer, Wintes und Iaena, Z. anorg. Chem. 25 (1900), 16.

- Gontz und Férée, Bull. Soc. Chim. 15 (1896), 834.

4 Mex, Zeitschr. phys. Chem. 29 (1899), 119. 
besitzen, ein Maximum der Schmelztemperatur zu bilden, das bedeuterd höher liegt als die Schmelztemperaturen der Komponenten und stets derselben Zusammensetzung $\mathrm{RHg}_{2}$ - wo $\mathrm{R}=\mathrm{Na}, \mathrm{K}$, Cs entspricht. So ist beim Systeme $\mathrm{Na}+\mathrm{Hg}$ von Kunxakow ${ }^{1}$ und spüter vou SCBULLLER ${ }^{2}$ die Verbindung $\mathrm{NaHg}_{z}$ ermittelt worden, welche bei $360^{\circ}$ schmilzt. Beim Systeme $\mathrm{K}+\mathrm{Hg}$ fanden ferner KunNakow und nacher $\mathrm{JäNECKE}^{3}$ die Verbindung $\mathrm{KHg}_{2}$, deren Schmelzpunkt bei $279^{\circ}$ liegt; beim Systeme $\mathrm{Cs}+\mathrm{Hg}$ endlich erhielten Kunnakow und ŻukowskY ${ }^{4}$ die Verbindung $\mathrm{CsHg}_{2}$ mit dem Schmelzpunkt $208.2^{\circ}$.

Die erwähnten Arbeiten ergaben noch, dafs Natrium, Kalium und Cäsium eine erhebliche Anzahl bestimmter Verbindungen mit Quecksilber zu bilden vermögen; diese Verbindungen sind, auch ihrer Zusammensetzung nach, einander sehr ähnlich.

Indessen wird in keiner Arbeit, die sich auf das System $\mathrm{Li}+\mathrm{Hg}$ bezieht, auf die Existenz einer Verbindung $\mathrm{LiNg}_{2}$ hingewiesen; ebenso ist die Anzahl der Verbindungen, welche Lithium mit Quecksiber bildet, selbst nach der ausführlichsten Abhandlung von MEx, verhältnismälsig gering. Lithium steht in manchen Beziehungen, wie z. B. in der Löslichkeit einiger Salze, etwas abseits von den anderen Metallen der Alkaligruppe und ähnelt mebr dem Magnesium. Es war also von Interesse zu untersuchen, ob Lithium diese Eigenschaften auch als Metall behält.

In der vorliegenden Arbeit wurde die Methode der thermischen Analyse angewandt und als Mefsapparat diente das Registrierpyrometer von KuRNakow. ${ }^{5}$

Für die Temperaturen unterhalb $100^{\circ}$ wurde das Thermoelement Kupfer-Konstantan angewandt. Bei diesem Element entsprach im Temperaturintervalle von $+100^{\circ}$ bis $-80^{\circ} 1 \mathrm{~mm}$ der Galvanometerskala $0.6^{\circ}$.

Als Ausgangsmaterial zur Herstellung der Legierungen dienten die reinsten Lithium- und Quecksilberpräparate von KaHLBAUM.

1 N. KURAarow, Journ. russ. phys.-chem. Ges. 31 (1899), 927; Z. anorg. Chem. 23 (1900), 439.

2 ScuÜller, $Z$. anorg. Chem. 40 (1904), 385.

s JäNeCKe, Zeitschr. phys. Chem. 38 (1907), 245.

4. Kunnanow und G. Zunowsky, Ber. d. St. Petersb. Polytechn. Inst. 6 (1906), 221; Journ. russ. phys.-chem. Ges. 37 (1905), 998; Z. anorg. Chem. $52(1907), 416$.

${ }^{5}$ N. Kunnakow, Ber. d. St. Petersb. Polytechn. Inst. 1 (1904), 183; Journ. russ. phys.-chem. Ges. 36 (1904), 841; Z. anorg. Chem. 42 (1904), 184. 
Die Analyse ergab die vollständige Abwesenheit von Schwermetallen und von Kalium im Lithium. Die spektroskopische Untersuchung zeigte Spuren von Natrium.

$\mathrm{Da}$ bei der Bildung von Lithium-Quecksilberamalgamen eine erbebliche Wärmeentwicklung stattfindet, so konnten weder Glasnoch Porzellangefälse benutzt werden, weil hierbei die Kieselsäure der Gefälse mit Lithium in Reaktion tritt.

Aus demselben Grunde konnte kein Glas- oder Porzellanrohr als Hülle für das Thermoelement verwendet werden, denn die Hülle, und auch das Thermoelement wurden ron der Schmelze, namentlich der lithiumreichen, vollständig zerstört.

Ich bediente mich daher eines Eisengefälses von $5 \mathrm{~cm}$ Höhe, $2,5 \mathrm{~cm}$ innerem Durchmesser und $5 \mathrm{~mm}$ Wandstärke. Eisen wird durch Lithium selbst bei Rotglut fast nicht verändert.

Das Gefäfs liefs sich vermittels eines besonderen Deckels, der auf Bolzen befestigt war, hermetisch verschliefsen. Bei Kontrollversuchen, durch Bestimmung des Schmelzpunktes des Bleis, ergaben sich sowohl in Eisen- wie auch in Porzellanhüllen identische Resultate.

Da sowohl die Zugabe von Quecksilber zu geschmolzenem Lithium wie auch die Zugabe von geschmolzenem Lithium zu Quecksilber starkes Spritzen zur Folge hatte, wodurch Verluste entstanden, und da ferner das Lithium hierbei oxydiert werden konnte, so wurde bei der Herstellung der Legierung in folgender Weise verfahren: Das aus Benzin herausgenommene, von der Kruste gereinigte Lithium wurde mit Filtrierpapier getrocknet und möglichst schnell in das Gefäls, in dem sich bereits etwas Quecksilber befand, hineingebracht und zwar so viel, dafs es das Gefäls füllte, wozu etwa $12 \mathrm{~g}$ Lithium erforderlich waren. Darauf wurde das Gefäls hermetisch verschlossen und dann erhitzt. Nachdem das Lithium geschmolzen und mit dem Quecksilber in Reaktion getreten war, was aus dem Erglühen des Gefälses zu erkennen war, wurde das letztere nach dem Erkalten geöffnet. Nach Bedarf wurde mitunter zu der geschmolzenen Masse eine gewisse Menge eines quecksilberreicheren Amalgams hinzugesetzt.

Unter solchen Bedingungen verlief die Reaktion ganz ruhig. Bei Anwendung flüssigen Paraffins (spez. Gew. 0.91) als Deckschicht schwimmen die Lithium-Quecksilberamalgame selbst bei einem Gehalte von 2 Atomprozent Quecksilber nicht auf der Oberfläche.

Um das Lithium vor Oxydation zu bewahren, war die An- 
Tabelle 1.

\begin{tabular}{|c|c|c|c|c|c|c|}
\hline \multirow{2}{*}{$\begin{array}{c}\text { Nr. } \\
\text { d.Ver- } \\
\text { suchs }\end{array}$} & \multirow{2}{*}{$\begin{array}{c}\text { Atom- } \\
0 i_{0} \\
\mathrm{Hg}\end{array}$} & \multirow{2}{*}{$\begin{array}{c}\text { Atom. } \\
0 / 0 \\
\mathrm{Li}\end{array}$} & \multicolumn{3}{|c|}{ Kristallisationstemperatur in ${ }^{\circ}$} & \multirow{2}{*}{ Anmerkung } \\
\hline & & & Beginn & $\begin{array}{l}\text { Umwand- } \\
\text { lungspunkt }\end{array}$ & $\begin{array}{l}\text { Eatek- } \\
\text { tikum }\end{array}$ & \\
\hline 1 & 一 & 100 & 179 & - & - & Punkt $A$ \\
\hline 2 & 2.4 & 97.6 & - & - & 162 & Eutektikum $B$ \\
\hline 3 & 4.6 & 95.4 & 207 & 166 & 162 & \\
\hline 4 & 6.7 & 93.3 & 226 & 166 & 162 & \\
\hline 5 & 7.3 & 92.7 & 232 & 166 & 162 & \\
\hline 6 & 8.8 & 91.2 & 250 & 166 & 162 & \\
\hline 7 & 9.3 & 90.7 & 253 & 166 & 162 & \\
\hline 8 & 10.0 & 90.0 & 260 & 166 & 162 & \\
\hline 9 & 12.7 & 87.3 & 265 & 166 & 162 & \\
\hline 10 & 13.7 & 86.3 & 270 & 166 & 162 & \\
\hline 11 & 14.0 & 86.0 & 272 & 166 & 162 & \\
\hline 12 & 16.4 & 83.6 & 275 & 166 & 162 & Imwandlgap. $C$ \\
\hline 13 & 18.0 & 82.0 & 315 & 166 & 162 & \\
\hline 14 & 21.2 & 78.8 & 348 & 166 & 162 & \\
\hline 15 & 21.9 & 78.1 & $35 \overline{5}$ & 166 & 162 & \\
\hline 16 & 23.1 & 76.9 & 364 & 166 & 162 & \\
\hline 17 & 23.4 & 76.4 & 369 & 166 & 162 & \\
\hline 18 & 24.8 & 75,2 & 376 & 166 & 162 & \\
\hline 19 & 24.9 & 75.1 & 879 & - & - & Punkt $D$ \\
\hline 20 & 25.6 & 74.4 & 379 & - & $\ldots$ & \\
\hline 21 & 26.2 & 73.8 & 379 & - & - & \\
\hline 22 & 27.3 & 72.7 & $3: 4$ & $\ldots$ & -- & \\
\hline 28 & 28.0 & 72.0 & 379 & - & - & \\
\hline 24 & 28.2 & 71.8 & 379 & - & - & \\
\hline 25 & 28.5 & $71 . \overline{5}$ & 379 & - & - & \\
\hline 26 & 29.9 & 70.1 & 379 & - & $\ldots$ & \\
\hline 27 & 30.9 & 69.1 & 379 & - & - & \\
\hline 28 & 31.1 & 68.9 & 379 & - & - & \\
\hline 29 & 34.7 & 65.3 & 406 & 379 & - & Punkt $E$ \\
\hline 30 & 36.9 & 63.1 & 440 & 379 & - & \\
\hline 31 & 37.8 & 62.2 & 453 & 379 & - & \\
\hline 32 & 38.2 & 61.8 & 464 & 879 & - & \\
\hline 33 & 38.8 & 61.2 & 478 & 379 & - & \\
\hline 34 & 39.5 & 60.5 & 490 & 379 & - & \\
\hline 35 & 39.9 & 60.1 & 496 & 379 & $\cdots$ & Punkt $S$ \\
\hline 36 & 42.3 & 57.7 & 534 & - & - & \\
\hline 37 & 45.0 & 55.0 & 564 & - & - & \\
\hline
\end{tabular}


Tabelle 1 (Fortsetzung).

\begin{tabular}{|c|c|c|c|c|c|c|}
\hline \multirow{2}{*}{$\begin{array}{c}\text { Nr. } \\
\text { d.Ver- } \\
\text { suchs }\end{array}$} & \multirow{2}{*}{$\begin{array}{c}\text { Atom- } \% \\
\mathbf{H} \\
0 / 0\end{array}$} & \multirow{2}{*}{$\begin{array}{c}\text { Atom- } 0 / 0 \\
\mathrm{Li} \\
0 / 0\end{array}$} & \multicolumn{3}{|c|}{ Kristallisationstemperatur in ${ }^{0}$} & \multirow{2}{*}{ Anmerkung } \\
\hline & & & Beginn & $\begin{array}{l}\text { Umwand- } \\
\text { lungspunkt }\end{array}$ & $\begin{array}{l}\text { Eutek- } \\
\text { tikum }\end{array}$ & \\
\hline 38 & 45.6 & 54.4 & 568 & - & - & \\
\hline 39 & 47.1 & 52.9 & 580 & - & $一$ & \\
\hline 40 & 47.4 & 52.6 & $579 . \overline{5}$ & - & - & \\
\hline 41 & 48.3 & 51.7 & 578.7 & - & - & \\
\hline 42 & 49.2 & 50.8 & 578.7 & - & - & \\
\hline 43 & 49.4 & 50.6 & 595 & - & - & \\
\hline 44 & 49.7 & ว0.3 & 597 & - & - & \\
\hline 45 & 49.9 & 50.1 & 600.8 & - & 一 & \\
\hline 46 & 50.0 & 50.0 & 600.5 & - & - & Distekt. Pkt. $F$ \\
\hline 47 & 50.4 & 42.6 & $\check{599}$ & - & - & \\
\hline 48 & 50.5 & 49.5 & 597 & - & $\ldots$ & \\
\hline 49 & 50.6 & 49.4 & 593 & $\ldots$ & 一 & \\
\hline 50 & 51.0 & 49.0 & 593 & - & - & \\
\hline 51 & 51.6 & 48.4 & 584 & - & - & \\
\hline 52 & 51.7 & 48.3 & 585 & - & - & \\
\hline 53 & 52.4 & 47.6 & 580 & - & - & \\
\hline 54 & 61.8 & 38.2 & 476 & 338 & - & \\
\hline 55 & 63.5 & 36.5 & 448 & 338 & - & \\
\hline 56 & 64.6 & 35.4 & 415 & 338 & - & \\
\hline 57 & 67.0 & 33.0 & 397 & 338 & 240 & \\
\hline 58 & 67.8 & 32.2 & 378 & 340 & 240 & \\
\hline 59 & 68.8 & 31.2 & 358 & 338 & 240 & \\
\hline 60 & 69.1 & 30.9 & 360 & 338 & 240 & \\
\hline 61 & 69.9 & 30.1 & 338 & 240 & - & Umwandlgp. $G$ \\
\hline 62 & 70.6 & 29.4 & 338 & 240 & - & \\
\hline 63 & 71.0 & 29.0 & 332 & 240 & - & \\
\hline 64 & 72.5 & 27.5 & 325 & 240 & - & \\
\hline 65 & 72.9 & 27.1 & 320 & 240 & - & \\
\hline 66 & 74.6 & 25.4 & 305 & 240 & - & \\
\hline 67 & 76.4 & 23.6 & 298 & 240 & -42 & Eutektikuın \\
\hline 68 & 78.4 & 21.6 & 276 & 232 & - & \\
\hline 69 & 78.7 & 21.3 & 270 & 232 & - & \\
\hline 70 & 79.5 & 20.5 & 261 & 232 & 一 & \\
\hline 71 & 80.1 & 19.9 & 256 & 232 & - & \\
\hline 72 & 80.2 & 19.8 & 249 & 232 & 一 & \\
\hline 73 & 80.6 & 19.4 & 247 & 232 & - & \\
\hline 74 & 80.7 & 19.3 & 246 & 232 & - & \\
\hline 75 & 80.9 & 19.1 & 242 & 232 & - & \\
\hline 76 & 81.3 & 18.7 & 238 & 232 & - & \\
\hline 77 & 81.6 & 18.4 & 234 & 232 & - & \\
\hline
\end{tabular}


Tabelle 1 (Fortsetzung).

\begin{tabular}{|c|c|c|c|c|c|c|}
\hline \multirow{2}{*}{$\begin{array}{c}\mathrm{Nr} . \\
\text { d.Ver- } \\
\text { suchs }\end{array}$} & \multirow{2}{*}{$\begin{array}{c}\text { Atom- } \\
\% \\
\mathrm{Hg}\end{array}$} & \multirow{2}{*}{$\begin{array}{c}\text { Atom- } \\
\% \\
\mathrm{Li}\end{array}$} & \multicolumn{3}{|c|}{ Kristallisationstemperatur in a } & \multirow{2}{*}{ Anmerkung } \\
\hline & & & Beginn & $\begin{array}{c}\text { Umwand- } \\
\text { lungspunkt }\end{array}$ & $\begin{array}{l}\text { Eutek- } \\
\text { tikum }\end{array}$ & \\
\hline 78 & 89.4 & 17.6 & 232 & - & -42 & Umwandlgp. $H$ \\
\hline 79 & 82.8 & 17.2 & 229 & - & - & \\
\hline 80 & 83.6 & 16.4 & 228 & & - & \\
\hline 81 & 85.8 & 14.2 & 216 & - & -42 & \\
\hline 82 & 86.9 & 13.1 & 203 & 一 & -42 & \\
\hline 83 & 88.8 & 11.2 & 184 & $\ldots$ & -42 & \\
\hline 84 & 89.3 & 10.7 & 173 & - & - & \\
\hline 85 & 90.9 & 9.1 & 160 & -- & - & \\
\hline 86 & 92.3 & 7.7 & 140 & -- & - & \\
\hline 87 & 92.5 & 7.5 & 132 & $\ldots$ & - & \\
\hline 88 & 92.8 & 7.2 & 128 & - & $-41 . \overline{5}$ & \\
\hline 89 & 94.2 & 5.8 & 110 & - & -41.5 & \\
\hline 90 & 97.5 & 2.5 & 11 & - & -42 & \\
\hline 91 & 99.03 & 0.97 & -30 & - & -42 & Eutektikum $K$ \\
\hline 92 & 100.00 & - & -39.6 & - & - & Punkt $L$ \\
\hline
\end{tabular}

wendung des Schmelzens in Wasserstoffatmosphäre nicht ratsam, weil man zu befürchten hatte, dals bei den hohen Schmelztemperaturen mancher Lithium-Quecksilberverbindungen die Bildung des stabilen Lithium-Wasserstoffs, - worauf bereits GcNTZ ${ }^{1}$ hingewiesen hat -, erfolgen könnte. Es wurde daher als Schutzschicht das eutektische Gemisch: 40.47 Atomprozent $\mathrm{KCl}+59.93$ Atomprozent $\mathrm{LiCl}$ verwendet, dessen Schmelzpunkt nach den Angaben von ŻemcáUżNy und RAMBaCH ${ }^{2}$ bei $352^{\circ}$ (nach GUNTZ $350^{\circ}$ ) liegt.

In den Fällen, bei denen die dem Erstarren des Metallgemisches entsprechenden Haltepunkte sehr nahe dieser Temperatur lagen, diente als Schutzschicht das eutektische Gemisch von 44.75 Atomprozent $\mathrm{RbCl}+55.25$ Atomprozent $\mathrm{LiCl}$, welches bei $312^{\circ}$ schmilzt.

Im Hinblick auf die Angabe von Guntz, ${ }^{1}$ dafs metallisches Lithium sich unter Bildung von Subchlorid in Chlorlithium lösen kann, wurde die Schutzschicht nach dem Gebrauche mehrmals analysiert; die Analyse ergab stets die Abwesenheit ron überschüssigem

${ }^{1}$ Guntz, Compt. rend. 123 (1895), 124. 244.

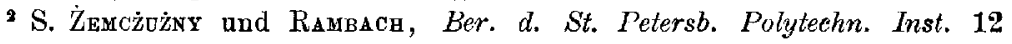
(1909), 340; Z. anorg. Chem. 65 (1910), 403. 
Lithium. Hieraus war zu schliefsen, dafs auch keine Oxydation des metallischen Lithium stattgefunden hatte, denn, hätte sich ein Oxyd gebildet, so wäre es in die Schutzschicht übergegangen.

Bei den niedrigschmelzenden Amalgamen diente als Schutzschicht flüssiges Paraffin.

Die Versuchsergebnisse sind in Tabelle 1 zusammengefafst und die graphische Darstellung der Änderungen der Schmelzpunkte des

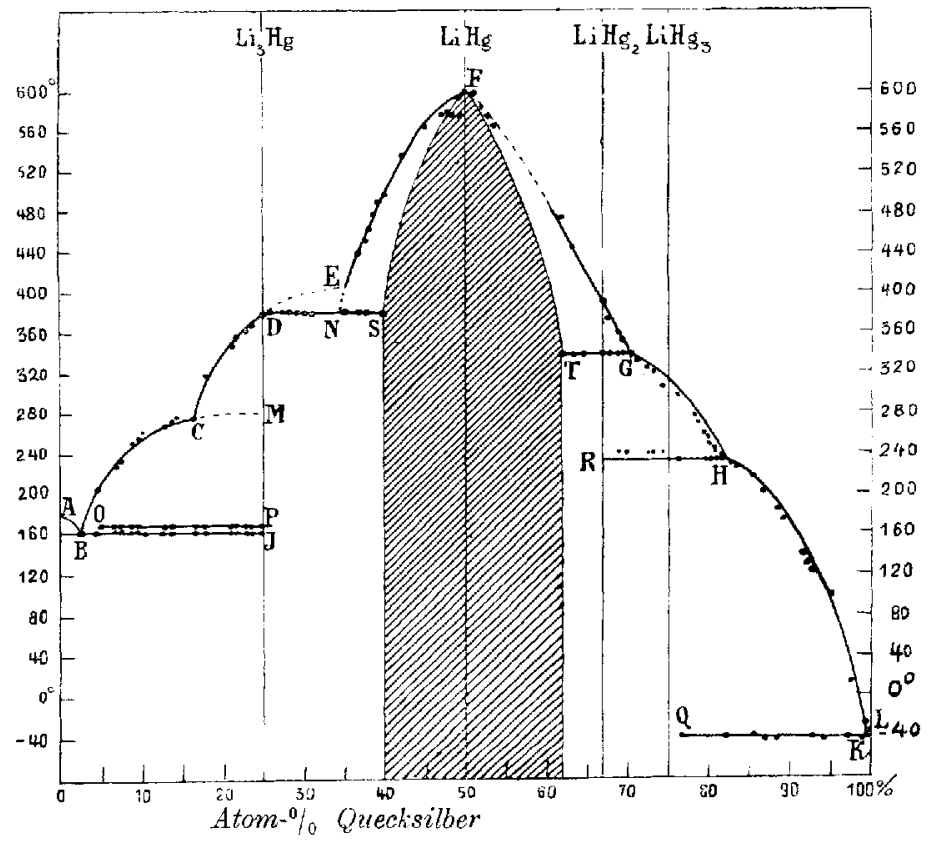

Fig. 1.

Systems $\mathrm{Li}+\mathrm{Hg}$ als Funktion der Zusammensetzung ist im Diagramme Fig. 1 wiedergegeben.

Das Diagramm ist so konstruiert, daf's auf der Abszissenachse die Atomprozente Quecksilber, auf der Ordinatenachse die Schmelzpunkte aufgetragen sind.

Wie aus Fig. 1 ersichtlich, besteht das Diagramm $A B C D E F G H K L$ aus einer Reibe von Ästen, die auf das Vorliegen einer ebensolchen Anzahl fester Phasen deuten. Die Bedingungen, unter denen die letzteren sich abscheiden, sind durch die folgenden besonderen Punkte charakterisiert.

Seiner allgemeinen Form nach unterscheidet sich das vorstehende Diagramm bedeutend von den entsprechenden Schmelz- 
Tabelle 2.

\begin{tabular}{|c|c|c|c|c|c|c|}
\hline \multirow{2}{*}{\multicolumn{4}{|c|}{ Besondere Punkte }} & \multicolumn{2}{|c|}{ Atomprozente } & \multirow{2}{*}{$\begin{array}{l}\text { Temperatur } \\
\text { in }\end{array}$} \\
\hline & & & & $\mathrm{Hg}$ & $\mathrm{Li}$ & \\
\hline Eutektischer Punkt $B$ & . & . & . & 2.4 & 97.6 & 162 \\
\hline Umwandlungspunkt $O$ & $\cdot$. & . . & . & 16.4 & 83.6 & 275 \\
\hline$" \quad E$ & . . & . . & . & 34.7 & 65.3 & 406 \\
\hline Dystektischer Punkt $F$ & . & . & . & 50.0 & 50.0 & 600.5 \\
\hline Umwandlungspunkt $G$ & . & . & . & 69.9 & 30.1 & 338 \\
\hline$" \quad H$ & . & . & . & 82.4 & 17.6 & 232 \\
\hline Eutektischer Punkt $K$ & . & . & . & $99.0-100$ & $0-1$ & -42 \\
\hline
\end{tabular}

kurven der Systeme $\mathrm{Na}+\mathrm{Hg}, \mathrm{K}+\mathrm{Hg}, \mathrm{Cs}+\mathrm{Hg}$. Während das Maximum der Schmelztemperaturen dieser Systeme der Zusammensetzung $\mathrm{RHg}_{2}$ - wo $\mathrm{R}=\mathrm{Na}, \mathrm{K}$, Cs - entspricht, liegt das Maximum des Systems $\mathrm{Li}+\mathrm{Hg}$ bei 50 Atomprozenten $\mathrm{Hg}$, was der $\mathrm{Zu}$ sammensetzung $\mathrm{LiHg}$ entspricht.

Bei näherer Betrachtung des vorliegenden Diagramms sehen wir, dafs die Schmelzkurve, beginnend von reinem Lithium, dessen Schmelzpunkt zu $179^{\circ}$ ermittelt worden ist, durch Zusatz von 2.4 A tomprozent Quecksilber bis auf $162^{\circ}$ herabgeht. Dieser Teil entspricht der Ausscheidung festen Lithiums in reinem Zustande und wird durch den Zweig $A B$ dargestellt. Die dem Punkte $B$ entsprechende Zusammensetzung stellt das eutektische Gemisch dar; es enthält 2.4 Atomprozente Quecksilber. Von diesem Punkte ab steigt die Schmelztemperatur und der $\mathrm{Z}_{\text {weig }} B C$ stellt die Ausscheidung der Krystalle der Verbindung $\mathrm{Li}_{3} \mathrm{Hg}$ dar, auf deren Existenz die eutektischen Haltepunkte bei $162^{\circ}$ hinweisen, die sich bei den Schmelzen bis zu 24.8 Atomprozent Quecksilber verfolgen lassen. Aufser diesen Haltepunkten weisen die Abkühlungskurven der Schmelzen im Intervalle von 2.4 bis zu 24.8 Atomprozent Quecksilber noch einen Haltepunkt bei $166^{\circ}$ auf; diese zuerst kaum bemerkbaren Haltepunkte werden immer ausgedehnter und erreichen bei 24.8 Atomprozent Quecksilber ein Maximum; das letztere deutet wahrscheinlich auf das Vorhandensein einer polymorphen Modifikation der Verbindung $\mathrm{Li}_{3} \mathrm{Hg}$ hin. Bei dem 16.4 Atomprozent Quecksilber entsprechenden Punkte $C$ der Schmelzkurve liegt ein Knick, was wohl darauf hinweist, dals bei weiterer Zugabe von Quecksilber sich eine neue Verbindung bildet, die unter Zersetzung schmilzt.

Die Zusammensetzung dieser Verbindung liels sich nicht genau feststellen. Die Schmelzkurve steigt beim Zusatz von Quecksilber 
aufwärts bis zu 25 Atomprozent Quecksilber (Punkt $D$ ), und dann treten bei konstanter Temperatur von $379^{\circ}$ Haltepunkte auf, die sich bis zu 39.9 Atomprozent Quecksilber verfolgen lassen. Die Vermutung der Existenz einer Verbindung $\mathrm{Li}_{3} \mathrm{Hg}_{2}$ wird jedoch durch den weiteren Verlauf des Zweiges $O D$ nicht bestätigt; der letztere nämlich verläuft nicht in der Richtung $D E$, wie es danach zu erwarten wäre, sondern erfährt im Punkte $D$ einen Knick und nimmt nachher die Richtung der Geraden DNS an. Es ist möglich, dafs die Krystallisationswärme der primären Krystalle dieser Verbindung so unerheblich ist, dafs sie vom Pyrometer nicht angezeigt wird. Die Länge (Dauer) der Haltepunkte nimmt zuerst von 25 bis zu 33 Atomprozent Quecksilber zu und dann bis 39.9 Atomprozent allmählich ab. Ein Zerfall in zwei Schichten wird hierbei nicht beobachtet. Es lassen sich somit aus der Betrachtung dieses Teiles der Kurve keine bestimmten Schlüsse auf die Zusammensetzung der sich ausscheidenden Phasen ziehen.

Bei etwa 33 Atomprozent Quecksilber steigt die Schmelzkurve steil aufwärts und erreicht den dystektischen Punkt $F$ bei $600.5^{\circ}$ bei einem Gehalte von 50.0 Atomprozent Quecksilber, was der stöchiometrischen Formel $\mathrm{LiHg}$ entspricht.

Bei weiterem Zusatz von Quecksilber fällt die Kurve. Es gelang bierbei unter gewissen Bedingungen Legierungen mit einem Gehalte bis zu 52.4 Atomprozent Quecksilber zu gewinnen. Von dieser Zusammensetzung ab bis zu 61.8 Atomprozent $\mathrm{Hg}$ wurden keine Legierungen des Lithiums mit Quecksilber erhalten, weil nämlich bei der hohen Schmelztemperatur der Verbindung $\mathrm{LiHg}$ die quecksilberreicheren Legierungen in LiHg und Quecksilber zerfallen. Letzteres destilliert über und es bleibt eine Legierung, die ihrer Zusammensetzung nach derselben Verbindung LiHg entspricht. Später jedoch gelang es bei den kalorimetrischen Bestimmungen auch in diesem Gebiete Legierungen $z u$ erbaiten, man mufs nur beim Zusatz der quecksilberreicheren Legierung zu der Verbindung LiHg das Gefäls hermetisch verschliefsen, dann erfolgt das Zusammenschmelzen unter dem Drucke der Quecksilberdämpfe.

Da durch die gewonnenen Ergebnisse der Charakter der Kurve in diesem Gebiete vollständig gekennzeichnet ist, so wurden keine Versuche an den Gemischen von 52.4-61.8 Atomprozent Quecksilber angestellt. Bei ihrem weiteren Verlauf fällt die Kurve immerfort bis zum Punkte $G$, der $338^{\circ}$ und einem Quecksilbergehalt von 61.8 Atomprozent entspricht. Die unteren Haltepunkte bei $338^{\circ}$ 
liefsen sich erst von 61.8 Atomprozent Quecksilber ab beobachten. A us der Tatsache, dals die unteren Haltepunkte links vom Maximum verschwinden, kann gefolgert werden, dafs die Verbindung $\mathrm{LiHg}$ im Gebiete von 39.9-61.8 Atomprozent $\mathrm{Hg}$ feste Lösungen sowohl mit Lithium wie auch mit Quecksilber zu bilden vermag.

Der Cmwandlungspunkt $G$ deutet auf die Bildung einer neuen festen Phase hin, deren Zusammensetzung sich aus den unteren Haltepunkten bei $232^{\circ}$, die man ron 67 Atomprozent Quecksilber ab verfolgen kann, zu $\mathrm{LiHg}_{2}$ ergibt. Der Ast $G H$ entspricht der Krystallisation dieser Verbindung.

Im Punkt $H$, der 82.4 Atomprozent Quecksilber entspricht, läfst sich ein Kuick beobachten und der Zweig $H G$ stellt die Krystallisationstemperatur einer neuen Verbindung dar, der die Zusammensetzung $\mathrm{LiHg}_{3}$ zuzuschreiben ist, weil die unteren eutektischen Haltepunkte bei $-42^{\circ}$ sich von einem Gehalte von 76.4 Atomprozent Quecksilber an verfolgen lassen.

Die Kurve $H K$ weist keine Knickpunkte auf, was auf das Fehlen irgendwelcher anderer Verbindungen des Lithiums mit Quecksilber hinweist; dieses widerspricht den Angaben anderer Forscher über die Existenz einer Verbindung $\mathrm{LiHg}_{5}$.

Der eutektische Punkt $K$ liegt zwischen 99.03 Atomprozent Quecksilber und reinem Quecksilber, und der Zweig $K L$ entspricht der Abscheidung des reinen Quecksilbers.

Es erweist sich somit als der am meisten charakteristische Teil des Diagrammes der Zweig $E F G$, der ein Temperaturmaximum $F$ besitzt: letzteres beweist unzweifelhaft die Existenz einer Verbindung $\mathrm{LiHg}$, die ohne Zersetzung bei $600.5^{0} \mathrm{schmilzt}$; diese Temperatur ist also bedeutend höher als die Schmelztemperatur der Komponenten dieses Systemes. Eine derartige Schmelzpunktserhöhung lälst sich, wie bekannt, unter den Gemischen der Alkalimetalle nur bei $\mathrm{Na}_{3} \mathrm{Bi}$, (Schmelzpunkt $775^{\circ},^{1}$ bei $\mathrm{Na}_{3} \mathrm{Sb} 856^{02}$ und bei $\mathrm{K}_{3} \mathrm{Bi}$ mit dem Schmelzpunkte $671^{\circ}$ ) beobachten. Im übrigen ist dies beim Lithium nicht der einzige Fall: viele seiner Verbindungen mit anderen Elementen sind sehr schwer schmelzbar. So schmilyt, nach Angaben von Guncz, ${ }^{4}$ die Verbindung LiH bei $680^{\circ}$, ferner schmilzt die ron

' Konnakow, Journ. russ. phys.-chem. Qes. 31 (1899), 927; Z. anorg. Chem. 23 (1900), 439. - Matuewson, Z. anorg. Chem. 50 (1906), 87.

2 Mathewson, $Z$. anorg. Chem. 50 (1906), 192.

' Sмiтн, Z. anorg. Chem. 56 (1907), 125.

- Guvrz, Compt. rend. 122 (1895), 244; 123 (1896), 694. 
Ocvrard ${ }^{1}$ und Guntz ${ }^{2}$ erhaltene Verbindung $\mathrm{Li}_{3} \mathrm{~N}$ noch nicht einmal bei Rotglut; das von LEBEA ${ }^{3}$ dargestellte $\mathrm{Li}_{3} \mathrm{Sb}$ schmilat erst oberbalb $950^{\circ}$ und endlich fanden Tammane und Masing den Schmelzpunkt von $\mathrm{Li}_{4} \mathrm{Sn}$ bei $680^{\circ}$.

Die Verbindung LiHg scheidet sich in würfelförmigen Kristallen $a b$ und unterscheidet sich von den anderen Verbindungen des Lithiums mit Quecksilber, die eine nadelartige Struktur aufweisen. Die Härte dieser Verbindung ist bedeutend höher als die der Komponenten und beträgt 2-3 nach der Skala von MoHs.

Aul'ser $\mathrm{LiHg}$ weisen die Äste $B C, C D E, G H$ und $H K$ auf noch vier Verbindungen hin, die unter Zersetzung schmelzen; so entspricht der Zweig $B C$ der Verbindung $\mathrm{Li}_{3} \mathrm{Hg}$, der Zweig $C D E$ $\mathrm{Li}_{n} \mathrm{Hg}_{2}$, wo $n=3$ oder 4 ; ferner entspricht der $Z$ weig $G H$ der Verbindung $\mathrm{LiHg}_{2}$ und der Zweig $H K$ endlich $\mathrm{LiHg}_{3}$.

Die Verbindangen $\mathrm{Li}_{3} \mathrm{Hg}$, $\mathrm{LiHg}$ und $\mathrm{LiHg}_{3}$ stimmen mit den Angaben von MeY vollkommen überein; die Verbindung $\mathrm{LiHg}_{2}$ ist ron ihm nicht angegeben worden. Um die Existenz dieser Ver. bindung zu bestätigen, wurden die Messungen der Bildungswärme von Gemischen mit einem Gehalt von 50-75 Atomprozent Quecksilber vorgenommen.

\section{Kalorimetrische Beobachtungen.}

Die Bestimmung der Bildungswärme der Li + Hg-Gemische wurde nach der von BERTHeLOT ${ }^{5}$ beim Studium der Bildungswärme von Natrium- und Kaliumamalgamen angewandten Methode aus. geführt.

Diese Methode besteht darin, dafs man den bei der Reaktion des betreffenden Gemisches + Säure gewonnenen kalorischen Effekt mit dem bei der Reaktion des reinen Alkalimetalls + Säure erhal. tenen vergleicht.

Als Säure diente hierfür Schwefelsäure von konstanter Konzentration, und zwar $1 \mathrm{Mol} . \mathrm{H}_{2} \mathrm{SO}_{4}$ auf 626.4 Mol. Wasser. Die Wärmekapazität dieser Säure wurde, gemäls den Angaben ron BIRoN ${ }^{\mathfrak{b}}$ zu

1 Ouvrarn, Compt. renul. 114 (1892), 120.

2 Gustz, Compt. rend. 123 (1895), 998.

${ }^{3}$ Lebeat, Compt. rend. 134 (1902), 231.

4 Masing und 'Tammane, Z. anorg. Chem. 67 (1910), 192.

5Berthelot, Ann. Chim. Phys. [5] 18 (1879), 433. 442.

E. Biron, Journ. russ. phys-chem. Ges. 1599, 171. 
0.990 angenommen: Die angewandte $\mathrm{Menge} \mathrm{H}_{2} \mathrm{SO}_{4}$ betrug bei allen Versuchen $501.4 \mathrm{~g}$ und der Wasserwert $496.39 \mathrm{cal}$.

Die Messungen wurden in einem geschlossenen Platinkalorimeter ausgeführt, dessen Gesamtwasserwert einschlielslich des Rührers und des eingetauchten Teiles des Thermometers 8.245 cal, betrug.

Das vorher präparierte Gemisch wurde beim Erstarren sorgfältig durchgerührt. Von diesem Amalgam wurde mittels eisernen Löffels eine gewisse Menge herausgenommen, in Benzin abgekühlt, von der anhaftenden Kruste gereinigt, mehrmals mit Benzin gewaschen und möglichst rasch in ein besonderes Glasgefäls (siehe Fig. 2) hineingebracht. Die Spureu von Benzin wurden

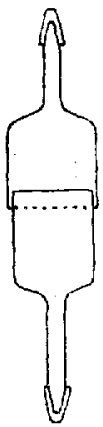

Fig. 2. durch einen Strom reinen trockenen Wasserstoff's verjagt; alsdann wurden auf die engen Röhrchen geschliffene Kappen aufgesetzt und das Gefäls gewogen. Das Gewicht des Amalgams ergibt sich aus der Differenz. Bei Benutzung eines solchen Apparates ist eine Oxydation des Alkalimetalles ausgeschlossen. Zur Kontrolle wurde die Kalorimeter-Flüssigkeit nach dem Versuche titriert und das Quecksilber gewogen. Während des Versuches wurde das Gläschen rasch geöffnet und das Amalgam ins Kalorimeter hineingeworfen.

Die Versuchstemperatur war $18-19^{\circ}$. Bei der Berechnung mulste eine Korrektion für die Schmelzwärme des Quecksilbers gemacht werden, die, nach PERson, ${ }^{1} 28.2$ cal. auf $1 \mathrm{~g}$ Quecksilber beträgt. Aufserdem mufste noch der Wärmeverlust, der durch infolge des entwickelten Wasserstoffs verdampfendes Wasser verursacht wird, in Rechnung gezogen werden. Diese Wärme beträgt nach THOMsLiN $^{2} 114$ cal. auf $1 \mathrm{~g}$ Wasserstoff.

Eine Korrektion für die Lösungswärme des gebildeten $\mathrm{Li}_{2} \mathrm{SO}_{4}$ war nicht erforderlich. Nach Thомsen beträgt dieselbe beim Lösen von $1 \mathrm{Mol} \mathrm{Li}_{2} \mathrm{SO}_{4}$ in $600 \mathrm{Mol} W$ asser 6050 cal. Da die Verdünnung bei unseren Versuchen bedeutend grölser war, so kann die Lösungswärme als proportional dem Betrag an metallischem Li gesetzt werden. Die Bildungswärme von LiOH.aq wurde in einem Vorversuch bestimmt, und zwar ergab sie sich zu 52.7 cal. (Mittelwert von 3 Bestimmungen). Diese Zahl kommt der von Guntz ${ }^{3}-53.2-$

\footnotetext{
1 Person, Pogg. Ann. 73 (1848), 471.

2 Thomsen, Thermochemische Untersuchungen III, S. 225.

3 Thomsen, Thermochemische Untersuchungen III. S. 199.227.
} 
ziemlich nahe und ist bedeutend gröfser als die von THоMSEx ${ }^{1}$ ermittelte, nämlich 49.1. Diese Abweichung läft sich wohl darauf zurückführen, dals das Lithium von THoMSEN unrein war. ${ }^{2}$

Ferner wurde die Bildungswärme der Reaktion $\mathrm{Li}+\mathrm{H}_{2} \mathrm{SO}_{4}+\mathrm{Ag}$ gemessen; die dabei erhaltenen Zahlen sind in Tabelle 3 zusammengestellt; der Mittelwert dieser Zahlen, $9635.3 \mathrm{cal}$. auf $1 \mathrm{~g} \mathrm{Li}$, ist den weiteren Rechnungen zugrunde gelegt. Die Bildungswärme eines Grammmol. $\mathrm{Li}_{2} \mathrm{SO}_{4}$ beträgt demnach $9635.3 \times 13.88=133.7 \mathrm{cal}$.

Die Messungsergebnisse der Gemische sind in der Tabelle 3 und Fig. 3 (Kurve 1) wiedergegeben. Die Zahlen sind auf je $1 \mathrm{~g}$ des Gemisches bezogen.

Tabelle 3.

\begin{tabular}{|c|c|c|c|c|c|c|c|c|c|c|c|}
\hline 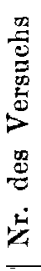 & 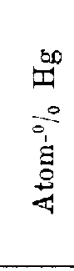 & $\begin{array}{l}3 \\
0 \\
0 \\
\vdots \\
\vdots \\
0 \\
0\end{array}$ & 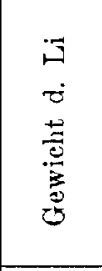 & 总 & 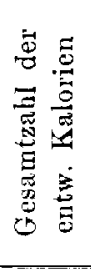 & 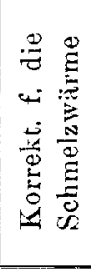 & 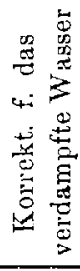 & 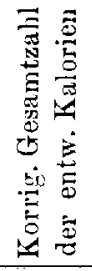 & 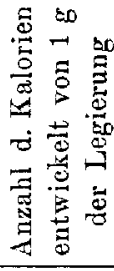 & 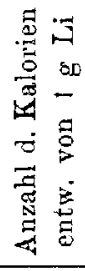 & 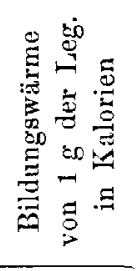 \\
\hline 1 & - & 100.0 & 0.1288 & - & 1232.3 & - & +2 & 1234.3 & - & - & $9573.5)$ \\
\hline 2 & - & 1000 & 0.1320 & - & 1278.1 & - & +2.1 & 1280.2 & - & - & or 9697.0$\}$ \\
\hline 3 & 50.67 & 49.33 & 0.1244 & 3.7657 & 824.5 & +10.3 & +2 & 836.8 & 318.0 & 222.2 & 96.8 \\
\hline 4 & 50.85 & 49.15 & 0.1488 & 4.5348 & 985.0 & +12.4 & +2.4 & 999.8 & 316.2 & 220.4 & 95.8 \\
\hline 5 & 61.63 & 38.37 & 0.1543 & 7.2164 & 927.5 & +19.9 & +2.5 & 949.9 & 206.0 & 131.6 & 74.4 \\
\hline 6 & 65.38 & 34.62 & 0.1275 & 6.9908 & 795 & +19.3 & +2 & 816.3 & 175.7 & 116.8 & 58.9 \\
\hline 7 & 68.49 & 31.51 & 0.1291 & 8.1264 & 774 & +22.6 & +2.1 & 798.3 & 153.1 & 98.3 & 54.8 \\
\hline 8 & 74.29 & 25.71 & 0.0976 & 8.1379 & 567.7 & +22.7 & +1.6 & 592.0 & 115.6 & 72.7 & 42.9 \\
\hline
\end{tabular}

Auf der Abszissenachse des Diagrammes sind die Atomprozente Quecksilber, auf der Ordinatenachse die Bildungswärmen, ausgedrückt in kleinen Kolonien auf je $1 \mathrm{~g}$ des Gemisches, aufgetragen.

Wie aus dem Diagramm ersichtlich, besteht die Kurve aus zwei Zweigen $A B$ und $B C$. Der Zweig $A B$ entspricht der Verbindung $\mathrm{LiHg}$, der $\mathrm{Z}$ weig $B C$ der Verbindung $\mathrm{LiHg}_{2}$, was aus dem Vorhandensein eines Knickes $B$ bei 66 Atomprozent Quecksilber hervorgeht.

${ }^{1}$ Guntz, Compt. rend. 123 (1896), 695. Siehe auch De Forcnand, Ann. Chim. Phys. [8] 9 (1906), 141, 235.

${ }^{2}$ Nach den Analysen von Ouvraind (l. c.) war die Zasammensetzung des reinsten Lithiumpräparates in damaliger Zeit folgende:

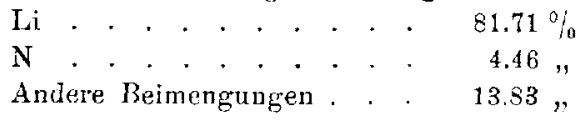


Zum Vergleich sind hier (Tabelle 4) die von Berthelot bei den Natrium- und Kaliumamalgamen erhaltenen Werte angeführt; letztere sind auf das $1 \mathrm{~g}$-Gewicht des Amalgams umgerechnet.

Wie ersichtlich, lassen die Knicke $G$ und $H$ (Fig. 3) der Kurve 2, die der Bildungswärme der Natriumamalgame entspricht, auf die

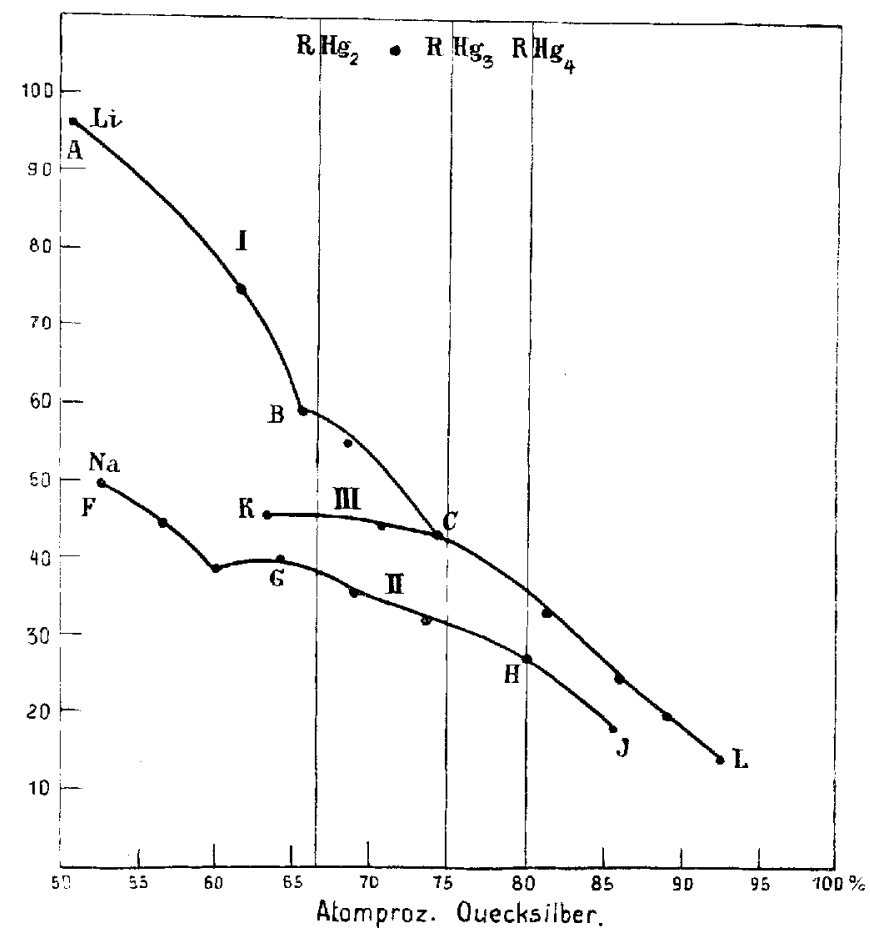

Fig. 3.

Existenz zweier Verbindungen $\mathrm{NaHg}_{2}$ und $\mathrm{NaHg}_{4}$ schliefsen, was auch durch die von KuRNakow und später von SchüLLER erhaltenen Schmelzdiagramme bestätigt worden ist.

Ebenso kann aus dem horizontalen Verlauf des Zweiges $K C$ und aus dem Knickpunkte $C$ der den Kaliumamalgamen entsprechenden Kurve 3 (Fig. 3) die Existenz der Verbindungen $\mathrm{KHg}_{2}$ und $\mathrm{KHg}_{3}$ gefolgert werden, was ebenfalls durch die Schmelzdiagramme von KURNAKOw und JäNECKE bestätigt wurde.

Demnach ist auch im vorliegenden Falle aus dem Vorhandensein von Knickepunkten auf der den Bildungswärmen von $\mathrm{Li}+\mathrm{Hg}$ entsprechenden Kurve auf die Ausscheidung einer neuen Phase zu schliefsen. 
Tabelle 4.

\begin{tabular}{|c|c|c|c|c|c|}
\hline Nr. & $\begin{array}{c}\text { Atom- } \% \\
\text { Hg }\end{array}$ & $\begin{array}{c}\text { Atom- } \% \\
\mathrm{Na}\end{array}$ & $\begin{array}{l}\text { Bildungswärme } \\
\text { v. Berthelor auf } \\
\text { auf } 1 \text { g-Atom Na } \\
\text { in grofsen Kal. }\end{array}$ & $\begin{array}{l}\text { Bildungswärme } \\
\text { angew. a. } 1 \mathrm{~g} \mathrm{~d} \text {. } \\
\text { Leg. in kleinen } \\
\text { Kalorien }\end{array}$ & $\begin{array}{l}\text { Zusammen- } \\
\text { setzung } \\
\text { der } \\
\text { Verbindung }\end{array}$ \\
\hline 1 & 52.7 & 47.3 & 12.1 & 49.2 & \\
\hline 2 & 56.6 & 43.4 & 12.7 & 44.7 & \\
\hline 3 & 600 & 40.0 & 12.5 & 38.7 & \\
\hline 4 & 64.3 & 35.7 & 15.3 & 39.9 & $\mathrm{NaHg}_{2}$ \\
\hline 5 & 69.0 & 31.0 & 16.8 & 85.8 & \\
\hline 6 & 73.7 & 26.3 & 18.7 & 32.0 & \\
\hline 7 & 80.0 & 20.0 & 22.3 & 27.0 & $\mathrm{NaHg}_{4}$ \\
\hline 8 & 85.7 & 14.3 & 21.6 & 17.6 & \\
\hline & & $\begin{array}{c}\text { Atom }-{ }^{\circ} \% \mathrm{~K} \\
36.7\end{array}$ & & & $\mathrm{KH}$ \\
\hline $\begin{array}{l}1 \\
2\end{array}$ & $\begin{array}{l}63.3 \\
70.6\end{array}$ & $\begin{array}{l}36.7 \\
29.4\end{array}$ & $\begin{array}{l}17.6 \\
23.0\end{array}$ & $\begin{array}{l}45.8 \\
44.3\end{array}$ & $\mathbf{K H} \mathrm{g}_{2}$ \\
\hline 3 & 74.0 & 26.0 & 26.2 & 43.0 & $\mathrm{KHg}_{3}$ \\
\hline 4 & 813 & 18.7 & 29.8 & 32.7 & \\
\hline 5 & 86.0 & 14.0 & 30.6 & 24.1 & \\
\hline 6 & 88.9 & 11.1 & 32.3 & 19.1 & \\
\hline 7 & 92.3 & 7.7 & 34.2 & 14.0 & \\
\hline
\end{tabular}

Somit wird die Existenz der Verbindung $\mathrm{LiHg}_{2}$ auch durch die kalorimetrischen Beobachtungen vollauf bestätigt.

Für die Existenz dieser Verbindung sprechen auch die Untersuchungen von Tammann und Masing ${ }^{1}$ über das System $\mathrm{Li}+\mathrm{Cd}$, bei dem sich ebenfalls eine Verbindung $\mathrm{LiCd}_{2}$ ergab.

Rechnet man die Bildungswärme von $\mathrm{LiHg}$ auf $1 \mathrm{Grammmol}$. Lithium um, so erhält man 20.2 cal., ein Wert, der sehr beträchtlich ist und der dem von $\mathrm{N}_{\mathrm{EBNST}}{ }^{2}$ für die Verbindung $\mathrm{HgO}=20.7 \mathrm{cal}$. sehr nahe kommt.

Auch die Verbindungen des Lithiums mit anderen Elementen bilden sich unter grofser Wärmeentwickelung; so beträgt die Bildungswärme von $\mathrm{LiH} 21.6$ cal. und die von $\mathrm{Li}_{3} \mathrm{~N} 16.5 \mathrm{cal}^{3}$ auf 1 Grammol. Lithium.

Tabelle 5 ergibt einen Vergleich über die Fähigkeit des Lithiums einerseits und des Magnesiums andererseits mit den Schwermetallen der unpaaren Reihen der II. Gruppe des periodischen Systems Verbindungen zu bilden.

Aus ihr geht hervor, dafs sämtliche Alkalimetalle und auch

1 l. c.

${ }^{2}$ Nernst, Zeitschr, phys. Chem. 2 (1888), 27.

${ }^{3}$ Guntz, ]. c. 
Tabelle 5.

\begin{tabular}{|c|c|c|c|}
\hline & $\mathrm{Zn}$ & $\mathrm{Cd}$ & $\mathrm{Hg}$ \\
\hline $\mathrm{Mg}$ & 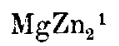 & $\mathrm{MgCd}^{1}$ & $\mathrm{MgHg}_{g}, \mathrm{MgHg}_{4}{ }^{2}$ \\
\hline $\mathrm{Ti}$ & - & $\mathrm{I}, \mathrm{iCd}, \mathrm{LiCd}_{2}$ & $\mathrm{Li}_{8} \mathrm{Hg}, \mathrm{LiHg}, \mathrm{LiHg}_{2}, \mathrm{LiHg}_{3}$ \\
\hline $\mathrm{Na}$ & $\mathrm{NaZn}_{11}^{3}$ & $\begin{array}{l}\mathrm{NaCd}_{2}, \mathrm{NaCd} \\
\mathrm{NaCd}_{9} ?^{4}\end{array}$ & $\begin{array}{c}\mathrm{Na}_{3} \mathrm{Hg}, \mathrm{Na}_{3} \mathrm{Hg}_{2}, \mathrm{Na}_{3} \mathrm{Hg}_{2}, \quad \mathrm{NaHg} \\
\mathrm{Na}_{19} \mathrm{Hg}_{13}, \mathrm{NaHg}_{g_{2}}, \mathrm{NaH}_{g_{4}}\end{array}$ \\
\hline $\mathbf{K}$ & $\mathrm{KZ} \mathrm{n}_{11}{ }^{5}$ & $\mathrm{KCd}_{7}, \mathrm{KCd}_{\mathbf{1 1}}{ }^{5}$ & $\begin{array}{c}\mathrm{KHg}, \mathrm{KHg}, \mathrm{KIIg}_{s}, \mathrm{KHg} g_{5} \text { oder } \\
\mathrm{KHg}_{6}, \mathrm{KH} \mathrm{H}_{10}\end{array}$ \\
\hline Cs & - & - & 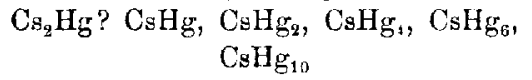 \\
\hline
\end{tabular}

Magnesium mit $\mathrm{Zn}$, $\mathrm{Cd}$ und $\mathrm{Hg}$ Verbindungen bilden, deren Anzahl mit steigendem Atomgewicht des Schwermetalls zunimmt. Quecksilber besitzt hiernach die grölste Fähigkeit, verschiedenartige Verbindungen mit den Alkalimetallen zu bilden; und zwar ist die Anzahl dieser Verbindungen bei Lithium am geringsten, ebenso bei Magnesium; auch ist die Zusammensetzung der Lithiumamalgame denen des Magnesiums sehr ähnlich.

Auf die Natur der $\mathrm{Mg}+\mathrm{Hg}$-Legierungen ist bis jetzt noch nicht näher eingegangen worden; indessen liegen einige Angaben über die Existenz einer Verbindung $\mathrm{MgHg}$ vor, der ein Schmelzpunktsmaximum zukommt. Ein Vergleich der Schmelzdiagramme der Systeme: $\mathrm{Li}+\mathrm{Cd}, \mathrm{Li}+\mathrm{Hg}$ and $\mathrm{Mg}+\mathrm{Cd}$ einerseits, bei denen die Verbindungen $\mathrm{LiCd}$, $\mathrm{LiHg}$ und $\mathrm{MgCd}$ sich als die am meisten charakteristischen erweisen, mit dem Systeme $\mathrm{Na}+\mathrm{Cd}$ andererseits, bei dem die Verbindung NaCd (siehe Tabelle 5) die charakteristische ist, lärst auf die Existenz der Verbindung $\mathrm{MgHg}$ a priori schlielsen.

Somit gelangt man zum Schlufs, dafs Iithium, auch im metallischen Zustande in manchen reinen Eigenschaften dem Magnesium mehr als den anderen Metallen der Alkaligruppe ähnelt.

1 Grube, Z. anorg. Chem. 49 (1906), 72. 77.

${ }^{2}$ Bachmetjew u. Wżarow, Journ. russ. phys.-chem. Ges. $2 \dot{0}$ (1893), 133.214.

${ }^{8}$ Mathewson, Z. anorg. Chem. 48 (1906), 196.

4 Kursakow und Kuswietzow, Journ. russ. phys.-chem. Ges. 31 (1899), 927; Z. anory. Chem. 23 (1900), 439, - Kurvakow, Journ. russ. phys.chem. Ges. 38 (1906), 809; Z. anorg. Chem. 52 (1907), 173. - Mathrwson, Z. anorg. Chem. $50(1906), 180$.

5 Swiтн, Z. anorg. Chem. 56 (1907), 1 i4. 119.

St. Petersburg, Polytechn. Institut Kaiser Peters des Gro/sen. Laboratorium für allgemeine Chemie.

Bei der Redaktion eingegangen am 2. Mai 1911. 